\title{
Rapid modeling of diffuse reflectance of light in turbid slabs
}

\author{
Lihong V. Wang \\ Optical Imaging Laboratory, Biomedical Engineering Program, Texas A\&M University, College Station, \\ Texas 77843-3120
}

Received September 10, 1997; revised manuscript received December 4, 1997; accepted December 8, 1997

An efficient and accurate hybrid model of the Monte Carlo technique and the diffusion theory was developed to simulate the diffuse reflectance of light in a turbid slab due to an infinitely narrow light beam. The narrow beam was normally incident on the top surface of the slab. The hybrid model was accurate in modeling the diffuse reflectance near the light source, where the diffusion theory was most inaccurate. The hybrid model was much faster than a pure Monte Carlo method by a factor as great as several hundred, depending on the optical properties, the thickness of the slab, and the settings of the hybrid and the Monte Carlo computations. The computation speed of the hybrid model was insensitive to the optical properties of the medium, in contrast to the pure Monte Carlo technique. The diffusion theory was accurate in modeling both the diffuse reflectance far from the source and the diffuse transmittance. The hybrid model and the diffusion theory should be used in conjunction for efficient and accurate computation of diffuse reflectance and diffuse transmittance. (C) 1998 Optical Society of America [S0740-3232(98)01004-7]

OCIS codes: $290.7050,290.1990,170.7050,120.5700,110.7050$.

\section{INTRODUCTION}

The study of applications of laser and incoherent light in biomedicine for both therapeutic and diagnostic purposes is an active research field. ${ }^{1,2}$ It is possible to treat lesions selectively without damaging the surrounding normal tissues by use of selective interactions between light and biological tissue. The selectivity, related to one or more of the characteristics of laser light, includes the wavelength selectivity of light absorption by certain tissue types where the monochromatic property of laser light is employed or the thermal or stress confinement selectivity where the short pulse width of laser light is used. It is also possible to diagnose disease noninvasively by use of the optical properties of biological tissues. The optical properties include index of refraction, absorption coefficient, scattering coefficient, and scattering anisotropy. It is believed that the spectra of the optical properties are related to the molecular conformation of biological tissues and hence should be sensitive indicators of the physiological status of tissues.

One of the fundamental goals in these studies is to understand and simulate light transport in biological tissues, which are turbid media. In these models the optical properties of biological tissues are the input parameters, and the light distribution in the tissues is calculated. Two types of method are usually used in the modeling. The first type starts from the radiative transfer equation, and the second uses the Monte Carlo technique. The radiative transfer equation is usually too complex to solve analytically and is often simplified by a diffusion approximation. Diffusion theory is quick but inaccurate in predicting the light distribution near the light source and boundaries. In many applications, especially the therapeutic applications, knowledge about the light distribution near the source is very important. The
Monte Carlo method is accurate but slow owing to its statistical nature.

A hybrid model of the Monte Carlo method and diffusion theory was developed to combine the accuracy advantage of the Monte Carlo method and the speed advantage of the diffusion theory for semi-infinite turbid media, ${ }^{3}$ which had a goal similar to that of the of the work of Flock et $a l .{ }^{4}$ but was different in the concept of implementation. Here the hybrid model is extended to model turbid slabs. The extended hybrid model is more useful in practice.

\section{METHOD}

The geometry and optical properties of a turbid slab can be described with four parameters. They include the thickness $d$, the relative refractive index $n_{\text {rel }}$, the absorption coefficient $\mu_{a}$, the scattering coefficient $\mu_{s}$, and the anisotropy factor $g$. The relative refractive index $n_{\text {rel }}$ is the ratio between the refractive indices of the turbid medium and the ambient medium. The absorption coefficient $\mu_{a}$ is defined as the probability of photon absorption per unit infinitesimal path length. The scattering coefficient $\mu_{s}$ is defined as the probability of photon scattering per unit infinitesimal path length. The anisotropy factor $g$ is the average cosine of the single scattered angle, where the scattering in tissue is often modeled by the Henyey-Greenstein scattering function. ${ }^{5,6}$

A cylindrical coordinate system is set up for this problem. The origin of the coordinate system is the point of light incidence on the top surface of the medium. The $z$ axis points downward into the turbid medium. The radial coordinate and azimuthal angle are denoted by $r$ and $\theta$, respectively.

\section{A. Diffusion Theory}

We present the diffusion theory for turbid slabs, using an extrapolated boundary condition, ${ }^{7}$ which is an extension 
to the theory for semi-infinite turbid media by Farrell et $a l{ }^{8}$ In the cylindrical coordinate system, the fluence at an observation point $(r, \theta, z)$ caused by an isotropic point source at $\left(r^{\prime}, \theta^{\prime}, z^{\prime}\right)$ in an infinite medium has been analytically solved:

$$
\phi_{1}\left(r, \theta, z ; r^{\prime}, \theta^{\prime}, z^{\prime}\right)=\frac{1}{4 \pi D} \frac{\exp \left(-\mu_{\mathrm{eff}} \rho\right)}{\rho},
$$

where $\rho$ is the distance between the observation point and the source point,

$$
\rho=\left[r^{2}+r^{\prime 2}-2 r r^{\prime} \cos \left(\theta-\theta^{\prime}\right)+\left(z-z^{\prime}\right)^{2}\right]^{1 / 2}
$$

$D$ is the diffusion constant

$$
D=\frac{1}{3\left[\mu_{a}+\mu_{s}(1-g)\right]},
$$

and $\mu_{\text {eff }}$ is the effective attenuation coefficient

$$
\mu_{\mathrm{eff}}=\sqrt{\mu_{a} / D}
$$

We are interested in the diffuse reflectance from the top surface of the slab and the diffuse transmittance from the bottom surface of the slab. The contribution of this isotropic point source to the diffuse reflectance is

$$
\begin{aligned}
R_{1}\left(r, \theta, 0 ; r^{\prime}, \theta^{\prime}, z^{\prime}\right) & =\left.D \frac{\partial \phi}{\partial z}\right|_{z=0} \\
& =\frac{z_{s}\left(1+\mu_{\mathrm{eff}} \rho\right) \exp \left(-\mu_{\mathrm{eff}} \rho\right)}{4 \pi \rho^{3}}
\end{aligned}
$$

where $\rho$ is the distance between the observation point $(r, \theta, 0)$ and the source point $\left(r^{\prime}, \theta^{\prime}, z^{\prime}\right)$. Similarly, the contribution of this isotropic point source to the diffuse transmittance is

$$
\begin{aligned}
T_{1}\left(r, \theta, d ; r^{\prime}, \theta^{\prime}, z^{\prime}\right) & =-\left.D \frac{\partial \phi}{\partial z}\right|_{z=d} \\
& =\frac{\left(d-z_{s}\right)\left(1+\mu_{\mathrm{eff}} \rho\right) \exp \left(-\mu_{\mathrm{eff}} \rho\right)}{4 \pi \rho^{3}},
\end{aligned}
$$

where $\rho$ is the distance between the observation point $(r, \theta, d)$ and the source point $\left(r^{\prime}, \theta^{\prime}, z^{\prime}\right)$. The diffuse reflectance and the diffuse transmittance in Eqs. (5) and (6) are applicable to a turbid slab only when the boundary condition is ignored.

To compute the fluence caused by an isotropic point source in a turbid slab based on Eq. (1), we need to convert the slab into an infinite medium by satisfying the boundary condition with an array of image sources. ${ }^{9}$ Since the fluence on the two real boundaries is not zero, two extrapolated virtual boundaries are used where the fluence is approximately zero. The two virtual boundaries are separated from the slab surfaces by a distance of $z_{b}$ (Fig. 1), where $z_{b}$ is

$$
z_{b}=2 A D
$$

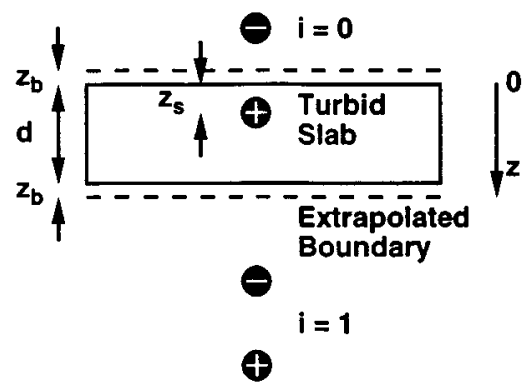

Fig. 1. Arrangement of the original and image point sources in a turbid slab, where the signs indicate whether a source is positive or negative.

where $A$ is related to the internal reflection $r_{i}$. When the boundary has matched refractive indices, $A=1$; otherwise, $A$ is estimated as ${ }^{10}$

$$
A=\left(1+r_{i}\right) /\left(1-r_{i}\right),
$$

where

$$
r_{i}=-1.440 n_{\text {rel }}^{-2}+0.710 n_{\text {rel }}^{-1}+0.668+0.0636 n_{\text {rel }} .
$$

Figure 1 shows the position of an original isotropic point source at $\left(r_{s}, \theta_{s}, z_{s}\right)$ and its image sources about the two virtual boundaries. Each mirroring about the virtual boundaries changes the sign of the point source. The $z$ coordinates of the $i$ th source pair are

$$
z_{s i \pm}=-z_{b}+2 i\left(d+2 z_{b}\right) \pm\left(z_{s}+z_{b}\right)
$$

where $i=0, \pm 1, \pm 2, \ldots$. When $i=0$, the source pair straddles the top boundary of the slab. When $i=1$, the source pair is the image of the 0th pair $(i=0)$ about the bottom extrapolated boundary. When $i=-1$, the source pair is the image of the first pair $(i=1)$ about the top extrapolated boundary. Although the number of image sources is infinite, the series may be truncated after several source pairs.

Once these image sources are used, the boundary condition is satisfied; hence the true boundaries can be removed. The original problem for the turbid slab is then converted into an array of isotropic sources of both signs in an infinite medium. A linear combination of Eq. (1) for the sources will therefore yield the fluence of the original isotropic point source in the tissue slab:

$$
\begin{aligned}
\phi\left(r, \theta, z ; r_{s}, \theta_{s}, z_{s}\right)= & \sum_{i=m}^{n}\left[\phi_{1}\left(r, \theta, z ; r_{s}, \theta_{s}, z_{s i+}\right)\right. \\
& \left.-\phi_{1}\left(r, \theta, z ; r_{s}, \theta_{s}, z_{s i-}\right)\right]
\end{aligned}
$$

where $m$ and $n$ are the lower and the upper limits of the truncated source pairs, respectively. A linear combina- 
tion of Eqs. (5) and (6) for the sources will yield the diffuse reflectance and the diffuse transmittance of the slab, respectively.

$$
\begin{aligned}
R\left(r, \theta, 0 ; r_{s}, \theta_{s}, z_{s}\right)= & \sum_{i=m}^{n}\left[R_{1}\left(r, \theta, 0 ; r_{s}, \theta_{s}, z_{s i+}\right)\right. \\
& \left.-R_{1}\left(r, \theta, 0 ; r_{s}, \theta_{s}, z_{s i-}\right)\right],
\end{aligned}
$$

$$
\begin{aligned}
T\left(r, \theta, d ; r_{s}, \theta_{s}, z_{s}\right)= & \sum_{i=m}^{n}\left[T_{1}\left(r, \theta, d ; r_{s}, \theta_{s}, z_{s i+}\right)\right. \\
& \left.-T_{1}\left(r, \theta, d ; r_{s}, \theta_{s}, z_{s i-}\right)\right] .
\end{aligned}
$$

If an infinitely narrow laser beam is incident upon a tissue slab, the narrow beam is approximated by an isotropic point source that is 1 transport mean free path $\left(L_{t}^{\prime}\right)$ below the tissue surface. Then the diffuse reflectance and the diffuse transmittance may be computed by use of Eqs. (12) and (13).

\section{B. Hybrid Model}

The key to using diffusion theory accurately was the precise conversion of the incident infinitely narrow photon beam into deep isotropic photon sources in the medium. ${ }^{11}$ Because of its high accuracy, Monte Carlo simulation is a good candidate to solve this conversion problem. The hybrid model consists of two steps: the Monte Carlo step and the diffusion step. The Monte Carlo step converts the incident photon beam into a distributed source term while escaped photons are collected. The diffusion step calculates the additional diffuse reflectance due to the source term by diffusion theory.

A critical depth $z_{c}$ is selected based on the transport mean free path of the turbid slab. When photons are within $z_{c}$ of the two boundaries of the slab, diffusion theory is considered inaccurate, and vice versa. Therefore, the Monte Carlo technique is used to trace photons until the photons reach the center zone that is $z_{c}$ from the two boundaries of the slab, where the $z$ coordinate of the center zone satisfies $z_{c} \leqslant z \leqslant d-z_{c}$ (Fig. 2).

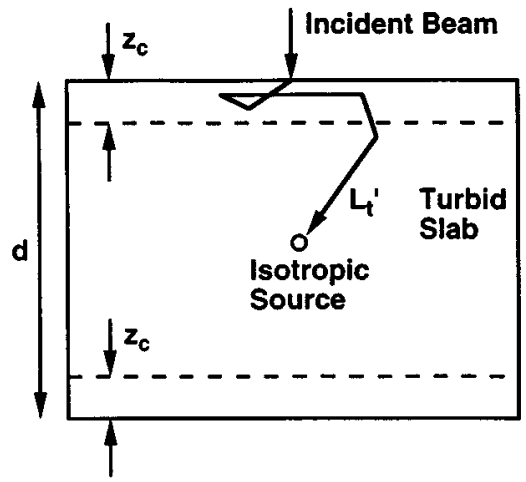

Fig. 2. Illustration of the conversion from an infinitely narrow light beam to an isotropic point source in the Monte Carlo step of the hybrid model. The last step of length $L_{t}^{\prime}$ converts the light into an isotropic point source.
The Monte Carlo step is based on the traditional Monte Carlo technique simulating light transport in tissue. ${ }^{12,13}$ Because the implicit photon-capturing technique ${ }^{14}$ is used during the Monte Carlo simulation, a photon packet with an initial weight of unity is launched perpendicularly to the surface along the $z$ axis (Fig. 2). If the boundary is index matched $\left(n_{\text {rel }}=1\right)$, all photon weight enters the turbid medium. Otherwise only a portion of the photon weight enters by means of Fresnel reflection. Then a step size $s$ is chosen statistically as

$$
s=\frac{-\ln (\xi)}{\mu_{a}+\mu_{s}},
$$

where $\xi$ is a random number equidistributed between 0 and $1(0<\xi \leqslant 1)$. The photon packet loses its weight partially at the end of each step because of absorption. The amount of weight loss is the photon weight at the beginning of the step multiplied by $(1-a)$, where $a$ is the albedo $\mu_{s} /\left(\mu_{a}+\mu_{s}\right)$. The photon with the remaining weight will be scattered. A new photon direction is statistically determined by the Henyey-Greenstein phase function according to the anisotropy factor $g$. A new step size is then generated by Eq. (14), and the process is repeated. When the photon weight is less than a preset threshold ( $10^{-4}$ in this study), Russian roulette is used to determine whether the photon should be terminated or continue propagation with an increased weight.

If the photon packet crosses the surface boundary into the ambient medium, the photon weight contributes to the diffuse reflectance $R_{\mathrm{mc}}(r)$ or the diffuse transmittance. If the photon packet reaches a position in the center zone that is $z_{c}$ from the two boundaries of the slab, the photon packet will be conditionally converted into an isotropic photon source after a new photon direction is statistically determined.

Similarity relations ${ }^{15,16}$ are invoked to convert the photon packet into an isotropic photon source. The similarity relations allow conversion from anisotropic scatterers into isotropic scatterers with a reduced scattering coefficient $\mu_{s}^{\prime}$, equal to $\mu_{s}(1-g)$, while the absorption coefficient is kept the same. The photon packet is moved a fixed step $L_{t}^{\prime}$ long, which is the mean free path for the converted isotropic scattering medium. If the new photon position is within $z_{c}$ of the two boundaries, the Monte Carlo simulation continues without the move of the $1-L_{t}^{\prime}$ step. If the new photon position is within the center zone that is $z_{c}$ from the two boundaries of the slab, the photon packet is converted into an isotropic point source. At the end of this step the photon packet interacts with the isotropic scattering medium according to an albedo $a^{\prime}$, equal to $\mu_{s}^{\prime} /\left(\mu_{a}+\mu_{s}^{\prime}\right)$. After absorption the new photon weight becomes the weight at the beginning of the step multiplied by the transport albedo $a^{\prime}$ and will experience scattering. After this scattering event, the photon becomes isotropic, and the weight of the photon packet is recorded into a source function $S(r, z)$, which is guaranteed to be zero within $z_{c}$ from the two slab boundaries. Multiple $(N)$ photon packets are traced to yield meaningful results because of the stochastic nature of the Monte Carlo simulation.

In the diffusion step, additional diffuse reflectance $R_{\text {diff }}(r)$ is calculated by use of the source term. Diffusion 
theory is used to compute the diffuse reflectance that is due to an isotropic point source, which is the impulse response. The recorded source function $S(r, z)$ is taken as the photon source, and these impulse responses weighted by the source function $S(r, z)$ are integrated to yield the diffuse reflectance $R_{\text {diff }}(r)$.

When the Monte Carlo step of the hybrid model is finished, the source function $S(r, z)$ gives the total photon weight in a grid element and may be converted into probability per unit volume $S_{d}(r, z)$, which is called source density. The diffuse reflectance due to the distributed source density $S_{d}(r, z)$ is calculated by

$$
\begin{aligned}
R_{\text {diff }}(r)= & \int_{0}^{\infty} \int_{0}^{\infty} \int_{0}^{2 \pi} S_{d}\left(r^{\prime}, z^{\prime}\right) R\left(r, 0,0 ; r^{\prime}, \theta^{\prime}, z^{\prime}\right) r^{\prime} \\
& \times \mathrm{d} \theta^{\prime} \mathrm{d} r^{\prime} \mathrm{d} z^{\prime}
\end{aligned}
$$

Because of its cylindrical symmetry, the diffuse reflectance $R_{\text {diff }}(r)$ is independent of the azimuthal angle $\theta$. Therefore we conveniently choose the observation point at $\theta=0$. The final diffuse reflectance will be the sum of the diffuse reflectances computed by the initial Monte Carlo step and the subsequent diffusion step:

$$
R_{d}(r)=R_{\mathrm{mc}}(r)+R_{\text {diff }}(r) .
$$

\section{Numerical Computation}

A grid system is set up on the cylindrical coordinate system to score the numerical quantities for this simulation. The grid lines are evenly spaced in the $r$ and $z$ directions. The number of grid elements in the $r$ and $z$ coordinates are $N_{r}^{\prime}$ and $N_{z}^{\prime}$, respectively. The grid separations in the $r$ and $z$ coordinates are $\Delta r^{\prime}$ and $\Delta z^{\prime}$, respectively. The center coordinates of the $i$ th $r$ grid element and $j$ th $z$ grid element are $r_{i}^{\prime}$ and $z_{j}^{\prime}$, respectively:

$$
\begin{aligned}
& r_{i}^{\prime}=(i+0.5) \Delta r^{\prime}, \\
& z_{j}^{\prime}=(j+0.5) \Delta z^{\prime} .
\end{aligned}
$$

At the end of the Monte Carlo step, $R_{\mathrm{mc}}(r)$ gives the total photon weight reflected into an annulus. We convert it into diffuse reflectance (probability per unit area) by dividing it by the total number of launched photon packets $(N)$ and the area of the annulus $\left(2 \pi r_{i}^{\prime} \Delta r^{\prime}\right)$.

The raw source function $S\left(r_{i}^{\prime}, z_{j}^{\prime}\right)$ is converted into the source density function $S_{d}\left(r_{i}^{\prime}, z_{j}^{\prime}\right)$ by

$$
S_{d}\left(r_{i}^{\prime}, z_{i}^{\prime}\right)=S\left(r_{i}^{\prime}, z_{i}^{\prime}\right) /\left(\Delta V_{i} N\right)
$$

where $\Delta V_{i}$ is the grid volume confined by the $i$ th $r$ grid element (an annulus) and the $j$ th $z$ grid element

$$
\Delta V_{i}=2 \pi r_{i}^{\prime} \Delta r^{\prime} \Delta z^{\prime}
$$

The grid system used to score the source term $S(r, z)$ is also used to compute the integration over $r^{\prime}$ and $z^{\prime}$ in Eq. (15). The symmetry of the integration over $\theta^{\prime}$ is used to lower the upper limit from $2 \pi$ to $\pi$. Therefore $R_{\text {diff }}(r)$ is computed from the following equation in terms of the source density $S_{d}(r, z)$.

$$
\begin{aligned}
R_{\mathrm{diff}}(r)= & \sum_{i=0}^{N_{r}^{\prime}-2} \sum_{j=0}^{N_{z}^{\prime}-2} S_{d}\left(r_{i}^{\prime}, z_{j}^{\prime}\right) r_{i}^{\prime} \Delta r^{\prime} \Delta z^{\prime} \\
& \times 2 \int_{0}^{\pi} R\left(r, 0,0 ; r_{i}^{\prime}, \theta^{\prime}, z_{j}^{\prime}\right) \mathrm{d} \theta^{\prime} .
\end{aligned}
$$

Note that the last grid elements in each direction are not used in the summation. Only a limited number of grid elements in each direction can be specified, but the step size for the Monte Carlo step has no bound because of the logarithmic operation [Eq. (14)]. Therefore the location of a photon packet may extend beyond the grid system. In this case we score the quantities into the last grid element in the direction of the overflow but do not use the last grid elements in the reflectance computation in Eq. (21). The truncation of this overflow gives a negligibly small error if the grid system is sufficiently large.

The integration over $\theta^{\prime}$ in Eq. (21) was done with Gaussian quadratures. ${ }^{17}$ The source density at some locations was much less than the maximum value. The contribution to the diffuse reflectance from the source density less than $1 \%$ of the peak source density was not computed to save computation time without significantly losing accuracy. The sum of $R_{\text {diff }}(r)$ and $R_{\mathrm{mc}}(r)$ yields the final diffuse reflectance $R_{d}(r)$. Both the pure Monte Carlo simulation and the hybrid model were implemented in ANSI (American National Standards Institute) Standard C. The programs are portable to any computer that supports ANSI Standard C. We conducted the computation on a Silicon Graphics Power Challenge computer running Irix 6.2 for this study.

The diffuse reflectance and the diffuse transmittance were computed by diffusion theory and the hybrid model and were compared with those computed by the pure Monte Carlo method, ${ }^{13,18}$ where the pure Monte Carlo results were considered accurate. Unless otherwise specified, 100,000 photon packets were used for this study in either the pure Monte Carlo method or the hybrid model.

\section{RESULTS}

Figure 3 shows the diffuse reflectance and the diffuse transmittance of an isotropic point source calculated by the pure Monte Carlo method ${ }^{13}$ and the diffusion theory described in the previous section. The isotropic point source was placed $L_{t}^{\prime}$ below the top surface of a slab. The pure Monte Carlo method was used to directly simulate the diffuse reflectance and the diffuse transmittance from this isotropic point source. In the diffusion theory calculation, one-three point-source pairs were employed. The locations and signs of the sources were shown in Fig. 1. The single-pair calculation was based on the pair of $i=0$. The double-pair calculation was based on the pairs of $i=0,1$. The triple-pair calculation was based on the pairs of $i=-1,0,1$.

Figure 4 shows the diffuse reflectance and the diffuse transmittance of an infinitely narrow light beam calculated by the pure Monte Carlo method and diffusion theory. The infinitely narrow light beam was normally incident upon the top surface of the slab. The Monte Carlo method directly simulated the response due to this 

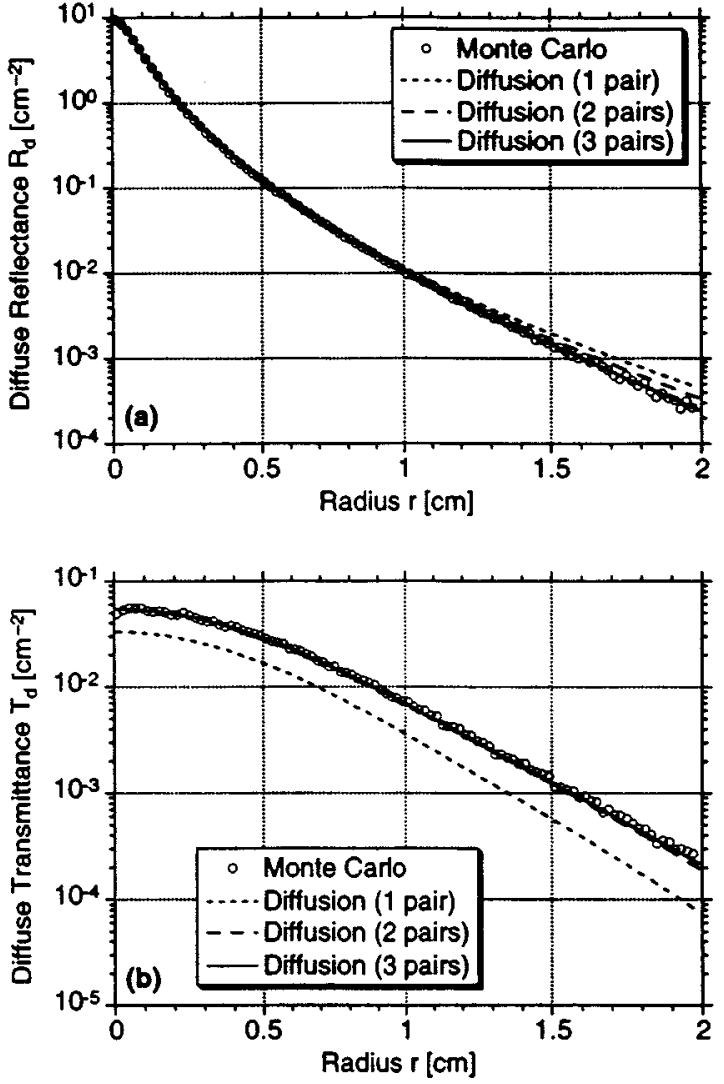

Fig. 3. Comparison between the pure Monte Carlo method and the diffusion theory in terms of (a) the diffuse reflectance and (b) the diffuse transmittance. The properties of the turbid slab included the relative index of refraction $n_{\text {rel }}=1$, the absorption coefficient $\mu_{a}=0.1 \mathrm{~cm}^{-1}$, the scattering coefficient $\mu_{s}=100 \mathrm{~cm}^{-1}$, the scattering anisotropy $g=0.9$, and the thickness $d=1 \mathrm{~cm}$.

narrow beam. In the diffusion theory calculation, the narrow beam was replaced with an isotropic point source located $L_{t}^{\prime}$ below the top surface of the slab. Three source pairs $(i=-1,0,1)$ were used for the diffusion theory calculation, where five of the six sources were the image sources of the point source at $z=L_{t}^{\prime}$. Figure 4(c) shows the relative errors of the diffuse reflectance and the diffuse transmittance calculated by the diffusion theory relative to those calculated by the pure Monte Carlo method. The relative errors were the difference between the results of the diffusion theory and the pure Monte Carlo method divided by the results of the pure Monte Carlo method.

Figure 5 shows the diffuse reflectance of an isotropic point source at various depths calculated by the pure Monte Carlo method and the diffusion theory. The isotropic point source was placed at $z=0.1 L_{t}^{\prime}, z=0.3 L_{t}^{\prime}$, and $z=0.5 L_{t}^{\prime}$. The pure Monte Carlo method was used to calculate the diffuse reflectance of these point sources individually. The diffusion theory was used to calculate the diffuse reflectance with three source pairs $(i=-1,0$, and 1). The relative errors in the diffuse reflectance for these three source locations between the diffusion theory and the Monte Carlo method are shown in Fig. 5(b), where the relative errors were defined as in Fig. 4(c).

Figure 6 shows the contours of the source density $S_{d}(r, z)$ calculated in the Monte Carlo step of the hybrid model. Figure 7 shows the diffuse reflectance of an infinitely narrow light beam calculated by the pure Monte Carlo method and the hybrid model. The infinitely narrow light beam was normally incident upon the top surface of the turbid slab. The pure Monte Carlo method directly simulated the diffuse reflectance of the narrow beam. The hybrid model converted the narrow beam into a source term as shown in Fig. 6, using the Monte Carlo technique while registering the diffuse reflectance. The hybrid model was then used to calculate the additional diffuse reflectance with the diffusion theory based on the source term as described in the previous section. The
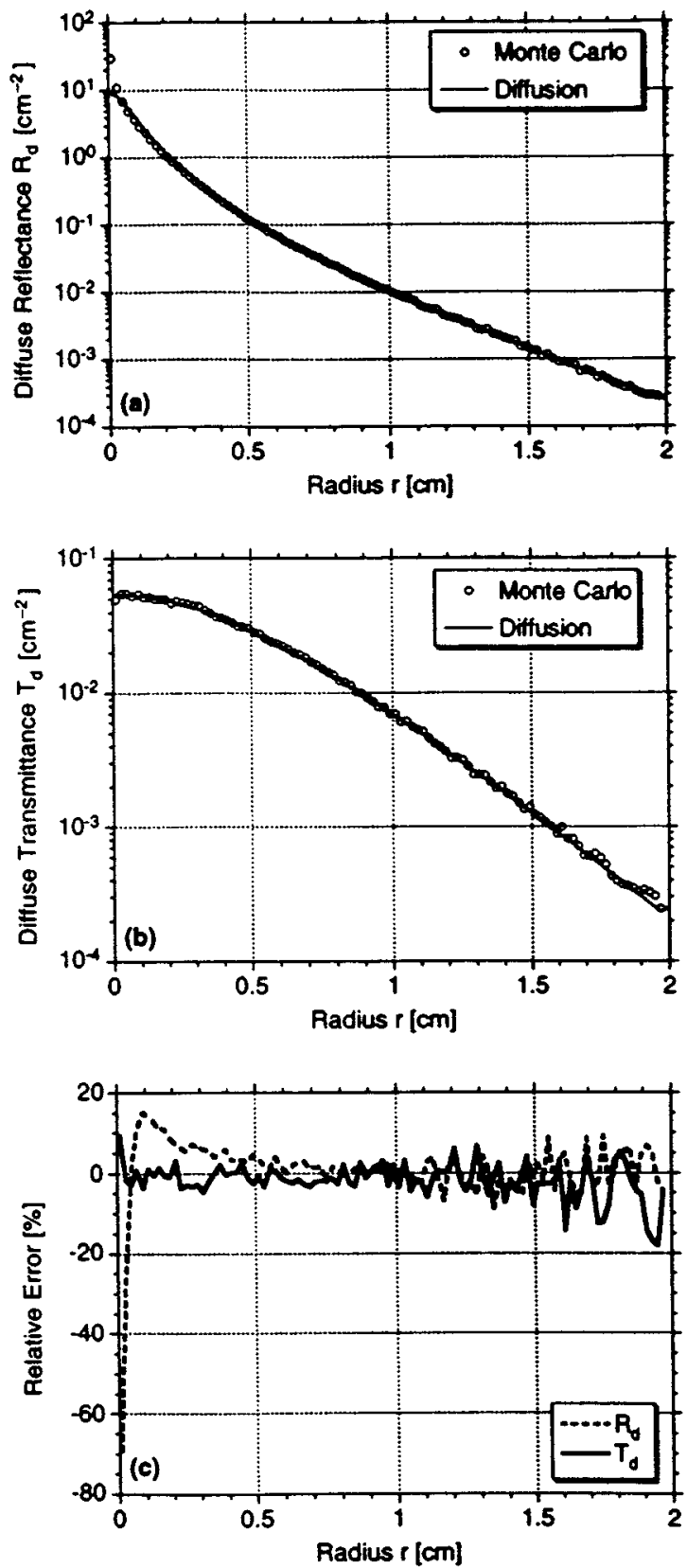

Fig. 4. Comparison between the pure Monte Carlo method and the diffusion theory in terms of (a) the diffuse reflectance and (b) the diffuse transmittance, and (c) the relative errors between the results. The properties of the turbid slab were described in Fig. 3. 

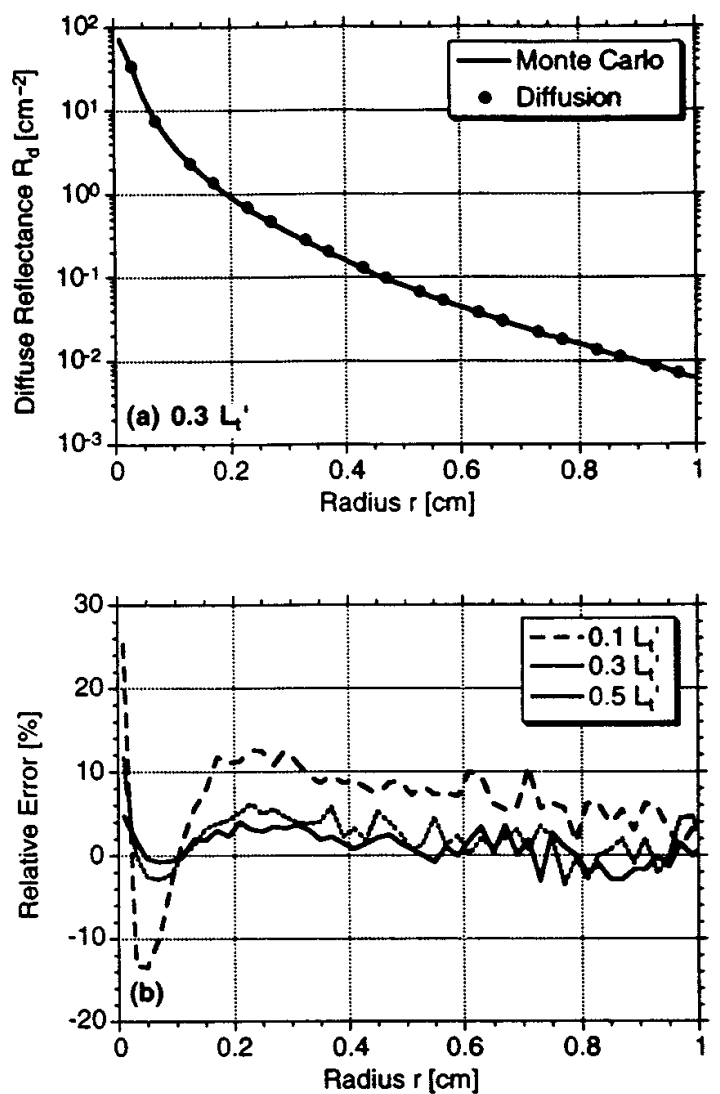

Fig. 5. (a) Comparisons between the pure Monte Carlo method and the diffusion theory in terms of the diffuse reflectance when an isotropic point source was placed at $z=0.3 L_{t}^{\prime}$ and (b) the relative errors between the results when an isotropic point source was placed at $z=0.1,0.3$, and $0.5 L_{t}^{\prime}$. The properties of the turbid slab were described in Fig. 3.

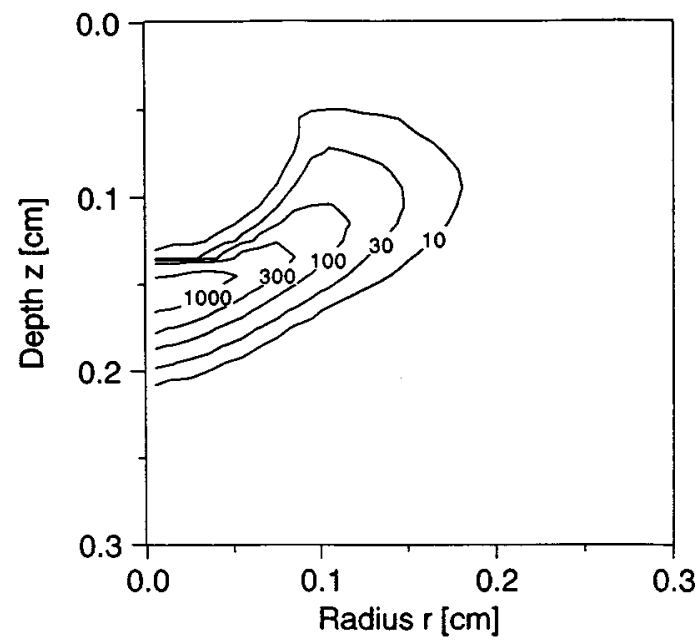

Fig. 6. Distribution of the source density generated by the initial Monte Carlo step in the hybrid model, where the critical depth $z_{c}$ was set to $0.05 \mathrm{~cm}$. The unit of the source density was in $\mathrm{cm}^{-3}$ for a unity input and would be in $W / \mathrm{cm}^{3}$ for a $1-W$ input. The properties of the turbid slab were described in Fig. 3.

critical depth $z_{c}$ was set to $0.05 \mathrm{~cm}$, which was approximately $0.5 L_{t}^{\prime}$. The number of grid elements in both the $r$ and the $z$ directions was 30. The size of grid elements in the $r$ and the $z$ directions was $0.01 \mathrm{~cm}$. Figure 7(b) plots the relative error in the diffuse reflectance between the two models. The relative error was defined as the difference between the diffuse reflectance calculated by the hybrid model and that calculated by the pure Monte Carlo method divided by the diffuse reflectance calculated by the pure Monte Carlo method. The relative error including the statistical variation was within $\pm 6 \%$.

The user time for the above pure Monte Carlo simulation was $4133.5 \mathrm{~s}$. The user time for the hybrid simulation was $170.6 \mathrm{~s}$, of which $166.5 \mathrm{~s}$ was spent in the initial Monte Carlo step, and the rest was spent in the diffusion step. The hybrid model was 24 times as fast as the Monte Carlo method for this simulation, where 1,000,000 photon packets were traced in both models.

Figure 8 shows the diffuse reflectance of an infinitely narrow light beam calculated by the pure Monte Carlo method and the hybrid model. The narrow beam was normally incident upon the top surface of a turbid slab that had a mismatched boundary $\left(n_{\text {rel }}=1.37\right)$. The number of grid elements in both the $r$ and the $z$ dimensions was 100. The sizes of grid elements in the $r$ and the $z$ directions were 0.005 and $0.003 \mathrm{~cm}$, respectively. The number of photon packets that were traced in the simulation was 100,000 . The user times of the pure Monte Carlo method were 697.5, 583.0, and $135.3 \mathrm{~s}$ for $\mu_{a}=0.1,1,10 \mathrm{~cm}^{-1}$, respectively. When the critical depth was set to $0.05 \mathrm{~cm}$, the user times of the hybrid model were $103.5,99.3$, and $76.2 \mathrm{~s}$ for $\mu_{a}=0.1,1$, and
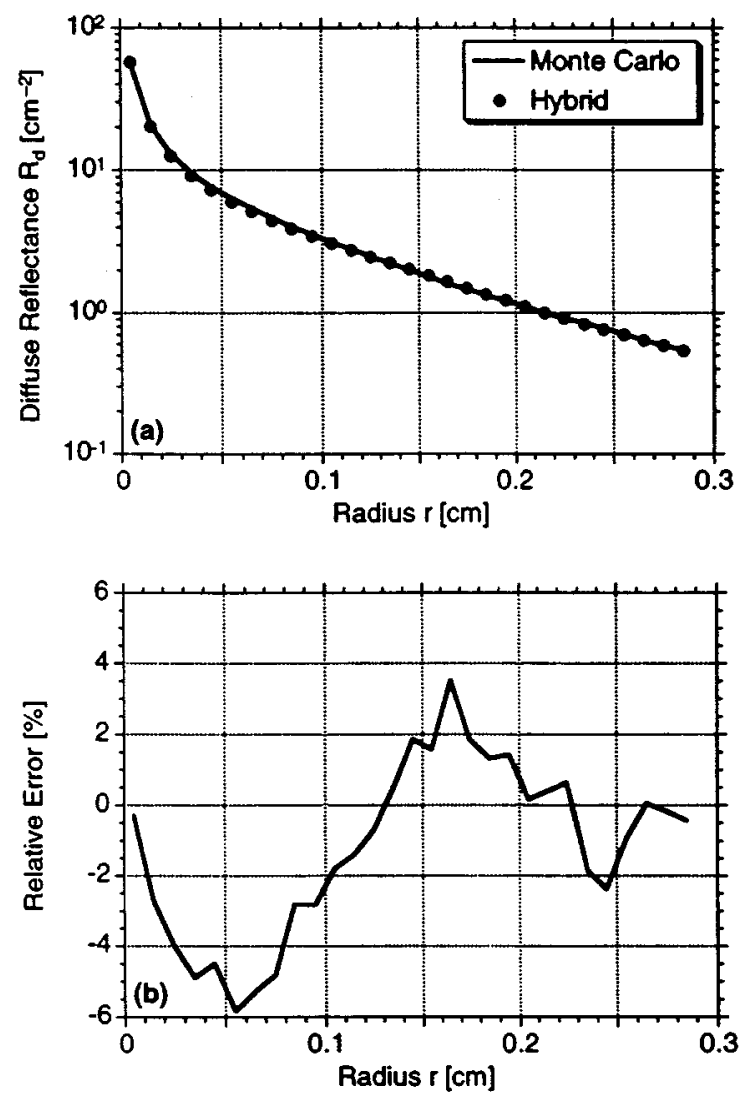

Fig. 7. (a) Comparisons between the pure Monte Carlo method and the hybrid model in terms of the diffuse reflectance and (b) the relative error between the results. The properties of the turbid slab were described in Fig. 3. 

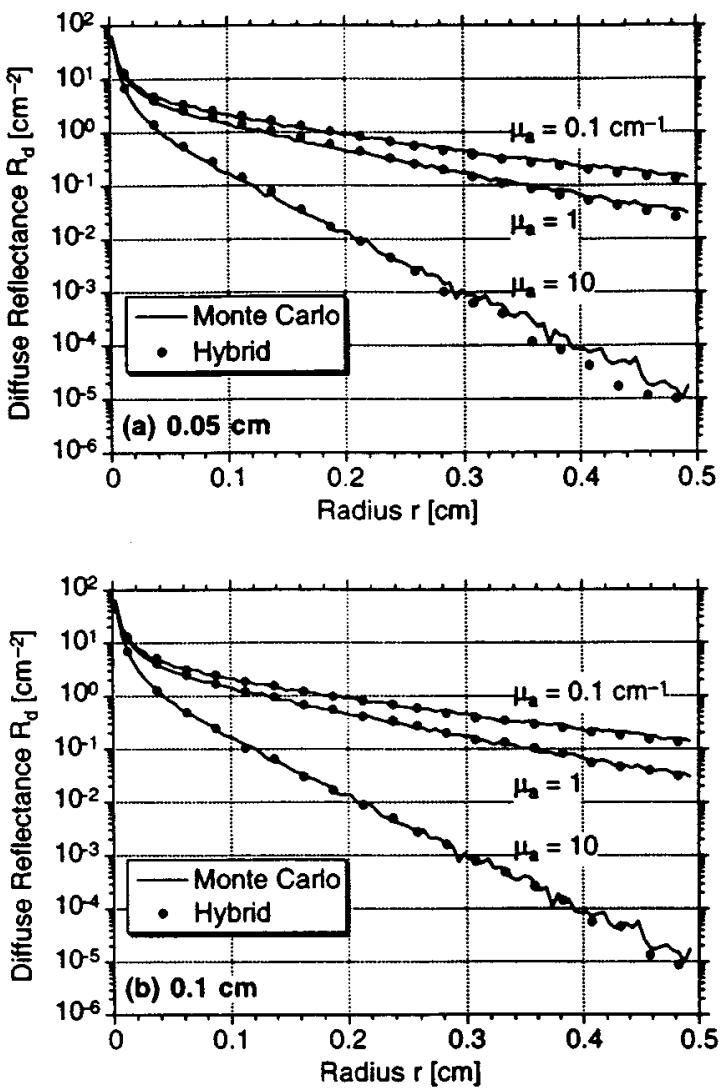

Fig. 8. Comparisons between the pure Monte Carlo method and the hybrid model in terms of the diffuse reflectance when the critical depth was set to (a) $0.05 \mathrm{~cm}$ and (b) $0.1 \mathrm{~cm}$. The absorption coefficient of the turbid slab was varied from $0.1,1,10 \mathrm{~cm}^{-1}$, while the other properties were held constant, including the relative index of refraction $n_{\text {rel }}=1.37$, the scattering coefficient $\mu_{s}$ $=100 \mathrm{~cm}^{-1}$, the scattering anisotropy $g=0.9$, and the thickness $d=1 \mathrm{~cm}$.
$10 \mathrm{~cm}^{-1}$, respectively. When the critical depth was set to $0.1 \mathrm{~cm}$, the user times of the hybrid model were 146.5, 141.7 , and $114.2 \mathrm{~s}$ for $\mu_{a}=0.1,1,10 \mathrm{~cm}^{-1}$, respectively.

Table 1 lists the user times of both the pure Monte Carlo method and the hybrid model for computations with various parameters. The absorption coefficient and the thickness of the turbid slab were varied while the other parameters were held constant. The critical depth $z_{c}$ was set to $0.05 \mathrm{~cm}$, which was approximately $0.5 L_{t}^{\prime}$. The number of grid elements in both the $r$ and the $z$ dimensions was 30 , where the grid size was $0.01 \mathrm{~cm}$. The number of photon packets that were traced in the simulations was 100,000 for both the pure Monte Carlo method and the hybrid model.

\section{DISCUSSION AND CONCLUSIONS}

The question of how many point-source pairs should be used in the diffusion theory to accurately model the diffuse reflectance and the diffuse transmittance was important. As shown in Fig. 3(a), three point-source pairs were required to achieve good accuracy in the diffuse reflectance within a 2 -cm radial distance from the source. No matter how many point-source pairs were used, the difference in the calculated diffuse reflectance close to the source was minimal and became large far from the source. As shown in Fig. 3(b), the zeroth pair did not give enough accuracy in calculating the diffuse transmittance. Two or three source pairs were required to achieve good agreement between the diffuse transmittance values that were calculated by the diffusion theory and those that were calculated by the pure Monte Carlo method. The number of point-source pairs that should be calculated would depend on the size of the observation region, the thickness of the slab, and the optical properties of the medium. In prac-

Table 1. User Times for the Pure Monte Carlo Method and the Hybrid Model and the Ratio between the User Times (Monte Carlo/Hybrid) under Various Conditions

\begin{tabular}{|c|c|c|c|c|c|}
\hline \multirow[b]{2}{*}{$n_{\text {rel }}$} & \multirow[b]{2}{*}{$d(\mathrm{~cm})$} & \multirow[b]{2}{*}{$\mu_{a}\left(\mathrm{~cm}^{-1}\right)$} & \multicolumn{2}{|c|}{ User Time (s) } & \multirow[b]{2}{*}{ Ratio } \\
\hline & & & Monte Carlo & Hybrid & \\
\hline 1.37 & 10 & 0.01 & 6684 & 23 & 291 \\
\hline 1.37 & 10 & 0.1 & 2589 & 23 & 113 \\
\hline 1.37 & 10 & 1.0 & 679 & 23 & 30 \\
\hline 1.37 & 3 & 0.01 & 2095 & 23 & 91 \\
\hline 1.37 & 3 & 0.1 & 1961 & 23 & 85 \\
\hline 1.37 & 3 & 1.0 & 679 & 23 & 30 \\
\hline 1.37 & 1 & 0.01 & 696 & 23 & 30 \\
\hline 1.37 & 1 & 0.1 & 698 & 23 & 30 \\
\hline 1.37 & 1 & 1.0 & 583 & 23 & 25 \\
\hline 1 & 10 & 0.01 & 3992 & 19 & 210 \\
\hline 1 & 10 & 0.1 & 1611 & 19 & 85 \\
\hline 1 & 10 & 1.0 & 468 & 19 & 25 \\
\hline 1 & 3 & 0.01 & 1253 & 19 & 66 \\
\hline 1 & 3 & 0.1 & 1201 & 19 & 63 \\
\hline 1 & 3 & 1.0 & 468 & 19 & 25 \\
\hline 1 & 1 & 0.01 & 415 & 19 & 22 \\
\hline 1 & 1 & 0.1 & 416 & 19 & 22 \\
\hline 1 & 1 & 1.0 & 382 & 19 & 20 \\
\hline
\end{tabular}

${ }^{a}$ The fixed optical properties included the scattering coefficient $\mu_{s}=100 \mathrm{~cm}^{-1}$ and the scattering anisotropy $g=0.9$. 
tice one may keep adding more image sources until the new sources make negligible addition to the calculated results.

Diffusion-theory-predicted diffuse reflectance was underestimated by a large margin (as great as 75\%) near the source compared with the accurate Monte Carlo result but became accurate far from the source [Figs. 4(a) and 4(c)]. As a rule of thumb, diffusion theory is accurate in modeling diffuse reflectance beyond a couple of transport mean free paths from the source and is accurate in modeling diffuse transmittance at all radii [Figs. 4(b) and 4(c)]. Therefore it would be efficient to use diffusion theory to calculate the diffuse reflectance far from the source and the diffuse transmittance and to use the hybrid model to calculate the diffuse reflectance near the source.

The choice of the critical depth would affect the computation accuracy and efficiency of the hybrid model. If the critical depth were too small compared with the transport mean free path, the computation error would be too large, although the computation would be fast and vice versa. As shown in Fig. 5, $0.3 L_{t}^{\prime}-0.5 L_{t}^{\prime}$ would be a good choice for the critical depth if a maximum of 5\%-12\% error were allowed, while $0.1 L_{t}^{\prime}$ would give a maximum error of $25 \%$.

The source term was centered at $z=0.15 \mathrm{~cm}$ as shown in Fig. 6. The selected critical depth was $0.05 \mathrm{~cm}$, which was close to $0.5 L_{t}^{\prime}$ since $L_{t}^{\prime}$ was $0.099 \mathrm{~cm}$. Once a photon reached the critical depth after a scattering event, it traveled $L_{t}^{\prime}$ further along the new direction after the scattering. If this new position of the photon was inside the center zone that was $z_{c}$ from the two boundaries of the slab, the photon was converted into an isotropic point source at the new position. Therefore the source term was densely populated near $z=1.5 L_{t}^{\prime}$. The source term was also limited to within approximately $2 L_{t}^{\prime}$ from the point of light incidence in both the $r$ and the $z$ dimensions.

The hybrid model had very good agreement with the pure Monte Carlo method (Fig. 7). The relative error including the statistical error was limited to $\pm 6 \%$. The statistical error may be further improved by use of more photon packets or enlargement of the grid size at the expense of reduced resolution. The systematic error may be further reduced by use of a larger critical depth at the expense of increased computation time. The hybrid model was significantly faster than the pure Monte Carlo method.

Under mismatched boundary conditions, the hybrid model had good agreement with the pure Monte Carlo method as well (Fig. 8). When the absorption coefficient $\mu_{a}$ increased to $10 \mathrm{~cm}^{-1}$ compared with the reduced scattering coefficient $\mu_{s}$ of $10 \mathrm{~cm}^{-1}$, the accuracy of the hybrid model became poor. Diffusion theory was valid only when $\mu_{a} \ll \mu_{s}^{\prime}$. Therefore the hybrid model was not expected to be accurate when this condition was not satisfied. When the critical depth was increased to $0.1 \mathrm{~cm}$, the accuracy of the hybrid model was improved even for the case of $\mu_{a}=10 \mathrm{~cm}^{-1}$ at the expense of increased computation time. The increased critical depth also caused a reduced contribution to the diffuse reflectance at the diffusion step of the hybrid model and made the Monte Carlo step dominant because the source density was expected to decrease. The accuracy of the hybrid model may be im- proved further by exploitation of an improved diffusion theory. ${ }^{19}$

The computation time of the hybrid model was insensitive to the optical properties (Table 1) unless the absorption coefficient became comparable with the reduced scattering coefficient (Fig. 8). In contrast, the pure Monte Carlo method was very sensitive to the optical properties. The lower the absorption, the longer the photon tracing because of the reduced chance of photon absorption per scattering event. It was also expected that the higher the scattering anisotropy, the longer the computation time, because the chance of photon termination as diffuse reflectance was reduced. The computation under the mismatched boundary condition took longer because photons could be internally reflected at the boundary and had an extended lifetime in the turbid slab. In all cases the hybrid model was significantly faster than the pure Monte Carlo method by a factor ranging from 20-291 in this study, depending on the optical properties, the slab thickness, the number of photons traced, the threshold photon weight for Russian roulette, and the choice of critical depth.

If the slab thickness becomes comparable with several transport mean free paths, diffusion theory will become inaccurate. In this case a pure Monte Carlo method should be used to calculate the diffuse reflectance and transmittance. Since the slab is optically thin, the computation time with the pure Monte Carlo method should be reasonably short. For example, it took only $74.5 \mathrm{~s}$ of user time for the response of a thin slab to be calculated by the pure Monte Carlo method. The properties of the slab included the relative index of refraction $n_{\text {rel }}=1$, the absorption coefficient $\mu_{a}=0.1 \mathrm{~cm}^{-1}$, the scattering coefficient $\mu_{s}=100 \mathrm{~cm}^{-1}$, the scattering anisotropy $g=0.9$, and the thickness $d=0.2 \mathrm{~cm}$. The user time increased to $136.3 \mathrm{~s}$ when the relative index of refraction $n_{\text {rel }}$ $=1.37$.

\section{SUMMARY}

The hybrid model is an efficient and accurate method in the calculation of diffuse reflectance of a turbid slab near the light source. The computation time of the hybrid model is insensitive to the optical properties of the slab in contrast to that of the pure Monte Carlo method. The hybrid model is many times faster than the pure Monte Carlo model. The critical depth in the hybrid model should be between $0.3 L_{t}^{\prime}$ and $0.5 L_{t}^{\prime}$ to achieve an accuracy of $\sim 10 \%$. The shorter the critical depth, the faster and less accurate is the computation and vice versa. Diffusion theory is accurate for calculation of both the diffuse reflectance that is a couple of transport mean free paths beyond the source and the diffuse transmittance. This work has potential applications in modeling of light transport in biological tissue, inverse algorithms of deducing optical properties, and image reconstruction algorithms.

\section{ACKNOWLEDGMENTS}

This project was sponsored in part by the National Institutes of Health grants R29 CA68562 and R01 CA71980, The Whitaker Foundation, and the Office of Vice President for Research at Texas A\&M University. 


\section{REFERENCES AND NOTES}

1. See related studies in S. L. Jacques, ed., Laser-Tissue Interaction VIII, Proc. SPIE 2975 (1997).

2. See related studies in B. Chance and R. R. Alfano, eds., $O p$ tical Tomography and Spectroscopy of Tissue: Theory, Instrumentation, Model, and Human Studies II, Proc. SPIE 2979 (1997).

3. L. -H. Wang and S. L. Jacques, "Hybrid model of Monte Carlo simulation diffusion theory for light reflectance by turbid media," J. Opt. Soc. Am. A 10, 1746-1752 (1993).

4. S. T. Flock, B. C. Wilson, and M. S. Patterson, "Hybrid Monte Carlo diffusion theory modeling of light distributions in tissue," in Laser Interaction with Tissue, M. W. Berns, ed., Proc. SPIE 908, 20-28 (1988).

5. S. L. Jacques, C. A. Alter, and S. A. Prahl, "Angular dependence of HeNe laser light scattering by human dermis," Lasers Life Sci. 1, 309-333 (1987).

6. L. G. Henyey and J. L. Greenstein, "Diffuse radiation in the galaxy," Astrophys. J. 93, 70-83 (1941).

7. L. -H. Wang, X. -M. Zhao, and S. L. Jacques, "Computation of the optical properties of tissues from light reflectance using a neural network," in Laser-Tissue Interaction $V$, S. L. Jacques, ed., Proc. SPIE 2134, 391-399 (1994).

8. T. J. Farrell, M. S. Patterson, and B. C. Wilson, "A diffusion theory model of spatially resolved, steady-state diffuse reflectance for the non-invasive determination of tissue optical properties in vivo," Med. Phys. 19, 879-888 (1992).

9. M. S. Patterson, B. Chance, and B. C. Wilson, "Time resolved reflectance and transmittance for the non-invasive measurement of tissue optical properties," Appl. Opt. 28, 2331-2336 (1989).

10. R. A. J. Groenhuis, H. A. Ferwerda, and J. J. Ten Bosch, "Scattering and absorption of turbid materials determined from reflection measurements. I: Theory," Appl. Opt. 22, 2456-2462 (1983).

11. L. -H. Wang and S. L. Jacques, "Analysis of diffusion theory and similarity relations," in Photon Migration and Imaging in Random Media and Tissues, B. Chance and R. R. Alfano, eds., Proc. SPIE 1888, 107-116 (1993).

12. S. A. Prahl, M. Keijzer, S. L. Jacques, and A. J. Welch, "A Monte Carlo model of light propagation in tissue," in Dosimetry of Laser Radiation in Medicine and Biology, G. J. Muller and D. H. Sliney, eds., Proc. SPIE IS 5, 102-111 (1989).

13. L. -H. Wang, S. L. Jacques, and L. -Q. Zheng, "MCMLMonte Carlo modeling of photon transport in multi-layered tissues," Comput. Methods Programs Biomed. 47, 131-146 (1995). The software package is downloadable from the web page at http://biomed.tamu.edu/ lw.

14. H. Kahn and T. E. Harris, "Estimation of particle transmission by random sampling," Monte Carlo Method, National Bureau of Standards Applied Mathematics Series No. 12 (U.S. Government Printing Office, Washington, D.C., (1951).

15. G. Yoon, S. A. Prahl, and A. J. Welch, "Accuracies of the diffusion approximation and its similarity relations for laser irradiated biological media," Appl. Opt. 28, 2250-2255 (1989).

16. D. R. Wyman, M. S. Patterson, and B. C. Wilson, "Similarity relations for anisotropic scattering in Monte Carlo simulations of deeply penetrating neutral particles," J. Comput. Phys. 81, 137-150 (1989).

17. W. H. Press, B. P. Flannery, S. A. Teukolsky, and W. T. Vetterling, Numerical Recipes in $C$, 2nd ed. (Cambridge U. Press, Cambridge, 1992).

18. L. -H. Wang, S. L. Jacques, and L. -Q. Zheng, "CONVConvolution for responses to a finite diameter photon beam incident on multi-layered tissues," Comput. Methods Programs Biomed. 54, 141-150 (1997).

19. A. Kienle and M. S. Patterson, "Improved solutions of the steady-state and the time-resolved diffusion equations for reflectance from a semi-infinite turbid medium," J. Opt. Soc. Am. A 14, 246-254 (1997). 